\title{
Diferenças na percepção da imagem corporal, no comportamento alimentar e no estado nutricional de universitárias das áreas de saúde e humanas
}

\author{
Differences in body image perception, eating behavior and nutritional status of college \\ students of health and human sciences
}

\author{
Maria Fernanda Laus ${ }^{1}$, Rita de Cássia Margarido Moreira ${ }^{2}$, Telma Maria Braga Costa ${ }^{3}$ \\ ${ }^{1}$ Mestre. Nutricionista. Doutoranda, Psicobiologia, Departamento de Psicologia e Educação, Faculdade de Filosofia, Ciências e Letras, Universidade de São Paulo (USP), \\ Ribeirão Preto, SP. ${ }^{2}$ Doutora. Psicóloga. Professora titular, Curso de Nutrição, Universidade de Ribeirão Preto (UNAERP), Ribeirão Preto, SP. ${ }^{3}$ Pós-Doutora. Nutricionista. \\ Professora titular e Coordenadora, Curso de Nutrição, UNAERP. \\ Trabalho realizado no Curso de Nutrição, Universidade de Ribeirão Preto (UNAERP), Ribeirão Preto, SP.
}

\section{Resumo}

Introdução: A literatura refere uma maior prevalência de transtornos alimentares em acadêmicas de cursos universitários nos quais a aparência física é importante, entre eles Educação Física e Nutrição. Supõe-se que pessoas preocupadas com seu peso e imagem corporal optem por essas áreas por terem um interesse pessoal pelo tema. O objetivo deste estudo foi avaliar a percepção da imagem corporal, o comportamento alimentar e o estado nutricional de estudantes de cursos das áreas da saúde e humanas para comparação entre estas.

Método: Aplicou-se o Eating Attitudes Test (EAT-26) e o Body Shape Questionnaire (BSQ) em 127 alunas do primeiro ano dos cursos de Nutrição e Educação Física (Saúde) e de Publicidade e Propaganda e Administração de Empresas (Humanas) que forneceram dados de peso e altura para cálculo do índice de massa corporal.

Resultados: Encontrou-se uma grande prevalência de distorção da imagem em todos os grupos, sem diferença entre as áreas ou entre os cursos. O EAT-26 indicou maiores escores nas estudantes da saúde em relação às de humanas, com alunas de Nutrição apresentando as maiores pontuações, estatisticamente diferentes das encontradas nos cursos de Publicidade e Administração, mas não de Educação Física. Além disso, a maioria das alunas foi classificada como eutrófica, e não se encontraram mulheres com obesidade em nenhum dos cursos, não havendo diferença significativa entre áreas ou cursos para esta variável.

Discussão: A alta incidência de distorção da imagem corporal associada à grande prevalência de comportamento alimentar inadequado nas alunas da área da saúde demonstra uma possível susceptibilidade ao desenvolvimento de transtornos alimentares.

Descritores: Imagem corporal, comportamento alimentar, estado nutricional, estudantes, transtornos da alimentação.

\begin{abstract}
Introduction: The literature reports a higher prevalence of eating disorders among undergraduate students whose majors value physical appearance, including Physical Education and Nutrition. It is possible to assume that people concerned about their weight and body image may choose to take these majors because they are personally interested in the topic. The objective of this study was to assess the body image perception, eating behavior, and nutritional status of students of health and human sciences and to compare these data.

Method: The Eating Attitudes Test (EAT-26) and the Body Shape Questionnaire (BSQ) were administered to 127 first-year students of Nutrition and Physical Education (health sciences) and Advertising and Business Administration (human sciences) who also provided information on weight and height for calculation of their body mass index.

Results: We found a high prevalence of body image distortion in all groups, with no difference between areas or majors. The EAT-26 indicated higher scores in students of health than in those who studied human sciences, with the students majoring in Nutrition showing the highest scores, with statistical difference from the scores found in Advertising and Business Administration, but not in Physical Education. In addition, most students were classified as eutrophic and there were not obese individuals in any of the majors, with no significant difference between areas or majors for this variable.

Discussion: The high incidence of body image distortion associated with high prevalence of inappropriate eating behavior in students of health sciences shows a possible susceptibility to the development of eating disorders.
\end{abstract}

Keywords: Body image, eating behavior, nutritional status, students, eating disorders.

Correspondência:

Telma Maria Braga Costa, Av. Costábile Romano, 2.201, Bloco U, Ribeirânea, CEP 14096-900, Ribeirão Preto, SP. Tel.: (16) 3603.6919. E-mail: tbraga@unaerp.br Não foram declarados conflitos de interesse associados à publicação deste artigo.

Copyright (C) Revista de Psiquiatria do Rio Grande do Sul - APRS

Recebido em 03/02/2009. Aceito em 16/07/2009. 


\section{Introdução}

O aumento da prevalência dos transtornos alimentares (TA) tem sido ressaltado nos últimos anos, e nas últimas duas décadas tem-se observado um aumento na frequência de problemas associados à imagem corporal e a comportamentos alimentares anormais entre os adolescentes ${ }^{1}$. A associação da beleza, sucesso e felicidade com um corpo mais magro tem levado as pessoas à prática de dietas abusivas e de outras formas não saudáveis de regular o peso ${ }^{2}$.

Esse aumento de prevalência dos TA, principalmente entre a população estudantil, tem levado inúmeros pesquisadores a intensificar seus estudos nesse campo de investigação em busca de um conhecimento mais aprofundado sobre as causas, a evolução, o tratamento, as possibilidades de recuperação desses quadros, bem como sobre as consequências dos TA para a vida social e educacional das pessoas acometidas por eles ${ }^{3}$.

Pessoas com TA têm em comum uma preocupação excessiva com peso e dieta, frequentemente apresentando distorção da imagem corporal, comportamentos alimentares anormais e práticas inadequadas para controle de peso ${ }^{4}$.

Estudos epidemiológicos têm demonstrado que as mulheres mais jovens constituem o grupo de maior risco para desenvolver esses transtornos, por serem mais vulneráveis às pressões dos padrões socioculturais, econômicos e esté$\operatorname{ticos}^{5,6}$.

A literatura refere, ainda, uma maior prevalência de TA em acadêmicas de cursos universitários nos quais a aparência física é importante ${ }^{7,8}$, entre eles o de Educação Física e Nutrição. Supõe-se que pessoas já preocupadas com o seu peso e imagem corporais podem optar por estas áreas justamente por já terem um interesse pessoal pelo tema ${ }^{9}$; entretanto, essa hipótese ainda é controversa ${ }^{7,8}$.

Portanto, o objetivo do presente estudo foi avaliar a percepção da imagem corporal, o comportamento alimentar e o estado nutricional de estudantes do primeiro ano de cursos vinculados às áreas de saúde e de humanas para posterior comparação entre estas.

\section{Método}

Este projeto foi aprovado pelo Comitê de Ética em Pesquisa em Seres Humanos da Universidade de Ribeirão Preto (UNAERP), Ribeirão Preto (SP). Trata-se de um estudo transversal realizado com todas as alunas do sexo feminino matriculadas no primeiro ano dos cursos de Nutrição $(n=24)$, Educação Física $(\mathrm{n}=37)$, Publicidade e Propaganda $(\mathrm{n}=32)$ e Administração de Empresas $(n=34)$ da UNAERP, na faixa etária de 18 a 22 anos de idade.

As investigações da percepção da imagem corporal e do comportamento alimentar foram realizadas por meio de questionários autoaplicáveis, respondidos individualmente.

A imagem corporal foi avaliada por meio do Questionário sobre a Imagem Corporal (Body Shape Questionnaire,
BSQ). Desenvolvido por Cooper et al. ${ }^{10}$ e traduzido e adaptado para o Brasil por Cordás \& Neves ${ }^{11}$, esse instrumento mede as preocupações com a forma do corpo, autodepreciação devido à aparência física e à sensação de estar "gordo"12. Cada questão é composta por seis alternativas de resposta, que variam do "sempre" ao "nunca". Para cada alternativa escolhida são conferidos pontos que variam de 1 a 6 (sempre $=6$; muito frequentemente $=5$; frequentemente $=4$; às vezes $=3$; raramente $=2$; nunca $=1$ ). O resultado do teste é a somatória dos 34 itens contidos no questionário, e a classificação dos resultados reflete os níveis de preocupação com a imagem corporal. Resultado menor que 70 é considerado um padrão de normalidade e tido como ausência de distorção da imagem corporal; resultados entre 70 e 90 pontos são classificados como leve distorção da imagem corporal; entre 91 e 110, como moderada distorção; e acima de 110 pontos a classificação é de presença de grave distorção da imagem corporal $^{13}$.

O comportamento alimentar foi avaliado por intermédio do Eating Attitudes Test (EAT-26). Este instrumento foi desenvolvido por Garner \& Garfinkel ${ }^{14}$, e sua versão reduzida foi proposta por Garner et al. ${ }^{15}$. A validação para o Brasil foi realizada por Bighett ${ }^{16}$, e o questionário rapidamente tornou-se o teste mais aplicado em estudos epidemiológicos para rastrear indivíduos supostamente susceptíveis ao desenvolvimento de TA, identificando casos clínicos em populações de alto risco e indivíduos com preocupações anormais em relação à alimentação e peso ${ }^{17}$. Cada questão é composta por seis alternativas de resposta que variam do "sempre" ao "nunca". Para cada alternativa escolhida são conferidos pontos que variam de 0 a 3 (sempre $=3$; muito frequentemente $=2$; frequentemente $=1$; às vezes $=0$; raramente $=0$; nunca $=0$ ). A única questão que apresenta pontos em ordem invertida é a 4, sendo que para as respostas mais sintomáticas, como "sempre", "muito frequentemente" e "frequentemente", não são dados pontos, e para as alternativas "às vezes", "raramente" e "nunca" são conferidos 1, 2 e 3 pontos, respectivamente. $\mathrm{O}$ resultado do teste é a somatória de todos os pontos dados aos 26 itens $^{18}$, sendo considerado um indicador de risco para o desenvolvimento de um TA o número de respostas positivas igual ou superior a $20^{19,20}$.

Adicionalmente à aplicação dos questionários, foi solicitado às estudantes que referissem seu peso e sua altura no momento do estudo para posterior classificação do estado nutricional.

A análise dos dados foi feita por meio das escalas de avaliação de cada um dos testes e do cálculo do índice de massa corporal (IMC), sendo utilizada a classificação do estado nutricional preconizada pela Organização Mundial de Saúde $(\mathrm{OMS})^{21}$.

Para o tratamento estatístico, utilizou-se o teste $t$ de Student para variáveis independentes na análise entre áreas e ANOVA na análise entre cursos, por intermédio do software Statistica, versão 5.0, com intervalo de confiança de 95\% (IC95\%). 


\section{Resultados}

Foram estudadas 127 universitárias, com distribuição da faixa etária variando entre 18 e 22 anos. Em relação à imagem corporal, observou-se uma grande prevalência de distorção intensa em todos os grupos (Tabela 1), e uma aná- lise estatística mais detalhada demonstrou que a distorção da imagem corporal não foi estatisticamente diferente entre as áreas de saúde e de humanas $(\mathrm{p}>0,05)$ (Tabela 2), ou entre os quatro cursos estudados $(p>0,05)$ (Tabela 3$)$, indicando que a distorção existe independente do curso ou da área de estudo.

Tabela 1 - Distribuição percentual das alunas de Nutrição, Educação Física, Publicidade e Propaganda e Administração de Empresas segundo as variáveis analisadas

\begin{tabular}{|c|c|c|c|c|}
\hline & Nutrição, \% & $\begin{array}{r}\text { Educação } \\
\text { Física, \% }\end{array}$ & $\begin{array}{c}\text { Publicidade } \\
\text { e Propaganda, \% }\end{array}$ & $\begin{array}{c}\text { Administração } \\
\text { de Empresas, \% }\end{array}$ \\
\hline \multicolumn{5}{|l|}{ BSQ } \\
\hline Sem distorção & 42 & 35 & 47 & 35 \\
\hline Leve & 12 & 19 & 22 & 30 \\
\hline Moderada & 21 & 16 & 3 & 9 \\
\hline Intensa & 25 & 30 & 28 & 26 \\
\hline Total & 100 & 100 & 100 & 100 \\
\hline \multicolumn{5}{|l|}{ EAT-26 } \\
\hline Positivo & 50 & 24 & 13 & 18 \\
\hline Negativo & 50 & 76 & 87 & 82 \\
\hline Total & 100 & 100 & 100 & 100 \\
\hline \multicolumn{5}{|l|}{ Estado nutricional } \\
\hline Desnutrição & 33 & 6 & 28 & 15 \\
\hline Eutrofia & 67 & 91 & 56 & 82 \\
\hline Sobrepeso & 0 & 3 & 16 & 3 \\
\hline Obesidade & 0 & 0 & 0 & 0 \\
\hline Total & 100 & 100 & 100 & 100 \\
\hline
\end{tabular}

BSQ = Questionário sobre a Imagem Corporal (Body Shape Questionnaire); EAT = Eating Attitudes Test.

Tabela 2 - Média \pm erro padrão dos resultados do Questionário sobre a Imagem Corporal, do Eating Attitudes Test e do índice de massa corporal referente às áreas de atuação avaliadas

\begin{tabular}{lcc}
\hline & Saúde & Humanas \\
\hline BSQ & $90,2 \pm 4,3$ & $82,4 \pm 4,0$ \\
EAT-26 & $16,6 \pm 1,1^{*}$ & $12,5 \pm 0,9$ \\
IMC & $20,2 \pm 0,2$ & $20,5 \pm 0,4$ \\
\hline
\end{tabular}

BSQ = Questionário sobre a Imagem Corporal (Body Shape Questionnaire); EAT = Eating Attitudes Test; IMC = índice de massa corporal. $* \mathrm{p}<0,05$.

Tabela 3 - Média \pm erro padrão da média dos resultados do Questionário sobre a Imagem Corporal, do Eating Attitudes Test e do índice de massa corporal referente aos cursos avaliados

\begin{tabular}{lcccc}
\hline & Nutrição & Educação & Publicidade & $\begin{array}{c}\text { Administração } \\
\text { de Empresas } \\
\text { e Propaganda }\end{array}$ \\
\hline BSQ & $90,6 \pm 6,6$ & $90,0 \pm 5,7$ & $81,5 \pm 5,6$ & $83,2 \pm 5,7$ \\
EAT-26 & $18,4 \pm 1,7^{*}$ & $15,3 \pm 1,3^{*}$ & $12,7 \pm 1,6$ & $12,3 \pm 1,25$ \\
IMC & $19,5 \pm 0,3$ & $20,7 \pm 0,3$ & $20,3 \pm 0,6$ & $20,7 \pm 0,4$ \\
\hline
\end{tabular}

BSQ = Questionário sobre a Imagem Corporal (Body Shape Questionnaire); EAT = Eating Attitudes Test; IMC = índice de massa corporal. $* \mathrm{p}<0,05$. 
Os resultados encontrados na análise do EAT-26 mostram uma alta incidência de positividade, especialmente nos cursos de Nutrição e Educação Física (Tabela 1). Encontrou-se uma diferença estatisticamente significante entre as áreas (Tabela 2), com as estudantes da área da saúde apresentando maiores escores quando comparadas às estudantes da área de humanas $(p=0,006)$. Além disso, a análise realizada entre os quatro cursos estudados demonstrou que as estudantes de Nutrição são as que apresentam as maiores pontuações nesse instrumento, sendo esses valores estatisticamente diferentes dos valores encontrados para os cursos de Publicidade e Administração, mas não de Educação Física $(p<0,05)$ (Tabela 3).

Quanto ao estado nutricional, observa-se na Tabela 1 que a maioria das estudantes foi classificada como apresentando IMC normal e não foram encontradas mulheres com obesidade em nenhum dos cursos. Para esta variável, as médias não foram diferentes estatisticamente entre as áreas da saúde e de humanas $(p>0,05)$ (Tabela 2), ou entre os cursos avaliados $(p>0,05)$ (Tabela 3$)$, indicando que o estado nutricional também é independente da futura área de atuação.

\section{Discussão}

As pesquisas nacionais referentes ao comportamento alimentar e à percepção da imagem corporal realizadas com estudantes universitárias se referem, geralmente, a alunas de Nutrição e Educação Física, porém poucos são os trabalhos que avaliam esses aspectos em alunas da área de Humanas.

Os achados referentes à percepção da imagem corporal demonstram que grande parte das mulheres estudadas apresenta algum grau de distorção da imagem corporal, independente do curso ou da futura área de atuação. Os resultados do presente estudo não corroboram dados da literatura, uma vez que a presença de distorção nesta amostra foi maior que a encontrada por outras pesquisas nacionais com universitárias.

Um estudo realizado por Pontieri et al..$^{22}$ com alunos de Educação Física, encontrou que 58,3\% das mulheres analisadas apresentaram ausência de distorção, e a distorção grave foi encontrada em $4,17 \%$ da amostra. Outro estudo, realizado por Stipp \& Oliveira ${ }^{2}$, que comparou a incidência de distúrbios do comportamento alimentar e da imagem corporal entre estudantes de graduação em Nutrição e Psicologia, concluiu que $54,8 \%$ das futuras nutricionistas e $51,1 \%$ das futuras psicólogas não possuem distorção da imagem, e a incidência de distorção intensa foi de 6,7 e 3,0\%, respectivamente.

$\mathrm{O}$ fato de não ter havido diferença estatisticamente significativa na percepção da imagem entre as áreas demonstra que distúrbios da imagem corporal acometem mulheres jovens em geral, e os dados relativos à presença de distorção entre as alunas dos quatro cursos estudados preocupam por haver prevalência de algum grau de distorção.

Este aspecto torna-se importante já que a percepção do peso corporal pode influenciar o comportamento alimentar, sendo que existem evidências de que distorções de imagem corporal ocorrem em maior frequência entre mulheres com $\mathrm{TA}^{23}$. Além disso, atualmente existe pouca dúvida quanto a que a supervalorização do peso parece ser um dos mais significativos fatores de risco para os comportamentos alimentares anormais e práticas inadequadas de controle de peso ${ }^{4}$.

Os resultados relativos à aplicação do EAT-26 nas estudantes de Educação Física, Publicidade e Administração corroboram dados da literatura ${ }^{22,24}$. Entretanto, observa-se que, no presente estudo, as estudantes de Nutrição mostraram um índice de positividade de $50 \%$, o que está em relativo desacordo com estudos relatados na literatura nacional pertinente, que demonstram percentuais de positividade entre 18 e $35 \%$ para estudantes desse curso ${ }^{2,25}$. Fiates \& Salles ${ }^{26}$, que compararam o comportamento alimentar de estudantes de Nutrição com o de estudantes de outros cursos desvinculados da área da saúde, chegaram a um percentual de formulários positivos ou $\geq 20(\mathrm{EAT}+)$ de 25,4 e $18,69 \%$, respectivamente; entretanto, essa diferença não foi estatisticamente significativa.

Observa-se, portanto, que os dados relativos à aplicação do EAT diferem dos demais trabalhos, uma vez que no presente estudo encontrou-se uma diferença significativa entre o comportamento alimentar de estudantes da área da saúde e de humanas.

Ainda não se sabe ao certo se o ambiente teria uma influência desencadeante ${ }^{6,27}$, ou se pessoas já predispostas a desenvolver TA tenderiam a procurar tais ocupações ${ }^{28}$. Uma possível explicação a ser considerada é a de que um padrão alimentar anormal poderia ser uma motivação pré-existente para a procura desses cursos ${ }^{8,9}$, e alguns autores afirmam que acadêmicos do primeiro ano geralmente apresentam maior risco de desenvolver um $\mathrm{TA}^{7,29}$, o que parece plausível no caso do presente estudo, já que as alunas entrevistadas ainda estavam cursando o primeiro semestre.

Além disso, um estudo realizado por Hughes \& Desbrow ${ }^{30}$ avaliando as motivações que levaram estudantes de Nutrição a escolher a carreira, encontraram que um dos principais fatores relatados foi uma experiência pessoal prévia com TA.

Em relação ao estado nutricional, diversos trabalhos têm realizado este levantamento em estudantes universitárias. Os dados relativos a esta variável demonstram que o índice de desnutrição entre as alunas de Nutrição e Publicidade e Propaganda foram mais altos que os encontrados por outros autores. Ressalta-se que, embora a utilização de peso e altura referidos seja uma prática pouco frequente no Brasil, possivelmente devido à carência de estudos nacionais e de base populacional analisando a validade de tais informações, em outros países, o estudo da validade do peso e da altura autorreferidos mostrou alta correlação entre os valores mensurados e aqueles autoinformados ${ }^{31,32}$.

Dados do estudo de Bosi et al. ${ }^{24}$ mostraram uma prevalência de $91 \%$ das futuras educadoras físicas dentro dos limites de normalidade. Já em relação às alunas de Nutrição, Penz et al. ${ }^{25}$ encontraram $75,8 \%$ das meninas desse curso dentro da faixa de normalidade.

Vários estudos demonstram que a maioria das universitárias brasileiras apresenta IMC normal ${ }^{22,24,25}$, resultados que corroboram 
os encontrados na presente pesquisa nas alunas de Educação Física e Administração. Já o elevado índice de desnutrição encontrado especialmente entre as alunas de Nutrição, embora não seja estatisticamente diferente dos índices encontrados nos demais cursos, não condiz com a literatura, uma vez que os valores encontrados são muito superiores aos relatados por outros autores.

Isso se torna particularmente preocupante no caso das futuras nutricionistas, já que elas pretendem trabalhar em promoção da saúde mediante o estímulo de hábitos alimentares saudáveis. Entretanto, alguns autores afirmam que, de maneira geral, estudantes de Nutrição em semestres mais avançados apresentam melhores hábitos alimentares que estudantes do primeiro ano, o que pode ser atribuído ao aumento do conhecimento na área'. Dessa forma, é de se esperar que, com o passar do curso, essas alunas passem a alimentar-se adequadamente, com consequente recuperação do estado nutricional.

Assim, como se pode observar, o presente estudo encontrou uma alta incidência de distorção da imagem corporal em todos os cursos, independente da área de atuação, bem como uma prevalência de eutrofia. No entanto, os resultados referentes ao comportamento alimentar demonstram uma tendência acentuada de anormalidade nos cursos da área de saúde.

É importante ressaltar que, apesar de não constituir causa primária da doença, a pressão para emagrecer em certas profissões, incrementada por expectativas de um bom desempenho, favorece a expressão de $\mathrm{TA}^{24}$.

\section{Conclusão}

A alta incidência de distorção da imagem corporal associada à grande prevalência de comportamento alimentar inadequado no grupo de alunas da área da saúde demonstra uma possível susceptibilidade dessas alunas ao desenvolvimento de TA. Por outro lado, apesar de o grupo de estudantes da área de humanas ter apresentado valores normais no EAT, os elevados índices de distorção da imagem corporal encontrados são preocupantes.

\section{Agradecimentos}

Os autores agradecem a colaboração da nutricionista Isadora Miranda Silva da Cruz pelo inestimável auxílio na coleta dos dados.

\section{Referências}

1. de Castro JM, Goldstein SJ. Eating attitudes and behaviors of pre- and postpubertal females: clues to the etiology of eating disorders. Physiol Behav. 1995;58(1):15-23.

2. Stipp LM, Oliveira MR. Imagem corporal e atitudes alimentares: diferenças entre estudantes de nutrição e de psicologia. Saude Rev. 2003;5(9):47-51.

3. dos Santos M, Meneguci L, de Mendonça AA. Padrão alimentar anormal em estudantes universitárias das áreas de nutrição, enfermagem e ciências biológicas. Ciencia et Praxis. 2008;1(1):1-4.

4. Nunes MA, Olinto MT, Barros FC, Camey S. Influência da percepção do peso e do índice de massa corporal nos comportamentos alimentares anormais. Rev Bras Psiquiatr. 2001;23(1):21-7.
5. Fairburn CG, Harrison PJ. Eating disorders. Lancet. 2003;361(9355):407-16.

6. American Dietetic Association. Position of the American Dietetic Association: nutrition intervention in the treatment of anorexia nervosa, bulimia nervosa, and eating disorders not otherwise specified (EDNOS). J Am Diet Assoc. 2001;101(7):810-9.

7. Reinstein N, Koszewski WM, Chamberlin B, Smith-Johnson C. Prevalence of eating disorders among dietetics students: does nutrition education make a difference? J Am Diet Assoc. 1992;92(8):949-53.

8. Fredenberg JP, Berglund PT, Dieken HA. Incidence of eating disorders among selected female university students. J Am Diet Assoc. 1996;96(1):64-5.

9. Korinth A, Schiess S, Westenhoefer J. Eating behavior and eating disorders in students of nutrition sciences. Public Health Nutr. 2009. [in press]

10. Cooper PJ, Taylor MJ, Cooper Z, Fairburn, CG. The development and validation of the body shape questionnaire. Int J Eat Disord. 1987;6(4):485-94.

11. Cordas TA, Neves JE. Escalas de avaliação de transtornos alimentares. Rev Psiq Clin. 1999;26(1):154-7.

12. Kakeshita IS. Estudo das relações entre o estado nutricional, a percepção da imagem corporal e o comportamento alimentar em adultos [dissertação]. Ribeirão Preto: Universidade de São Paulo; 2004.

13. Assunção SS, Cordás TA, Araújo LF. Atividade física e transtornos alimentares. Rev Psiquiatr Clin. 2002;19(1):4-13.

14. Garner DM, Garfinkel PE. The Eating Attitudes Test: an index of the symptom of anorexia nervosa. Psychol Med. 1979;9(2):273-9.

15. Garner DM, Olmsted MP, Bohr Y, Garfinkel PE. The eating attitudes test: psychometric features and clinical correlates. Psychol Med. 1982;12(4):871-8.

16. Bighetti F. Tradução e validação do Eating Attitudes Test (EAT-26) em adolescentes do sexo feminino na cidade de Ribeirão Preto [dissertação]. Ribeirão Preto: Universidade de São Paulo; 2003.

17. Vilela JE, Lamounier JA, Dellaretti Filho MA, Barros Neto JR, Horta GM. Transtornos alimentares em escolares. J Pediatr (Rio J). 2004;80(1):49-54.

18. Ribeiro RP. Indicadores de hábitos dietéticos e aspectos cognitivos e comportamentais relacionados aos distúrbios de conduta alimentar em adolescentes do sexo feminino [tese]. Ribeirão Preto: Universidade de São Paulo; 1999.

19. Nunes MA, Bagatini LF, Abuchaim AL, Kunz AR, Ramos D, Silva JA, Somenzi L, et al. Distúrbios da conduta alimentar: considerações sobre o Teste de Atitudes Alimentares (EAT). Rev ABP-APAL. 1994;16(1):7-10.

20. Magalhães VC, Mendonça GA. Transtornos alimentares em universitárias: estudo de confiabilidade da versão brasileira de questionários autopreenchíveis. Rev Bras Epidemiol. 2005;8(3):236-45,

21. World Health Organization (WHO). Obesity: preventing and managing the global epidemic. Geneva: WHO; 1998. p. 276.

22. Pontieri FM, Lopes PF, Eça VB. Avaliação da presença de fatores de risco para o desenvolvimento de transtornos alimentares em acadêmicos de um curso de Educação Física. Rev Cienc Biol Saude. 2007.

http://ww4.unianhanguera.edu.br/programasinst/Revistas/revistas2007/cienciasbesaude/Avaliacao_da_presenca_de_fatores.pdf. Acessado em: 03/12/2008

23. ter Bogt TF, van Dorsselaer SA, Monshouwer K, Verdurmen JE, Engels RC, Vollebregh WA. Body mass index and body weight perception as risk for internalizing and externalizing problem behavior among adolescents. J Adolesc Health. 2006;39(1):27-34.

24. Bosi ML, Luiz RR, Uchimura KY, de Oliveira FP. Comportamento alimentar e imagem corporal entre estudantes de Educação Física. J Bras Psiquiatr. 2008;57(1):28-33.

25. Penz LR, Bosco SM, Vieira JM. Risco para desenvolvimento de transtornos alimentares em estudantes de Nutrição. Scientia Med. 2008;18(3):124-8.

26. Fiates GM, de Salles RK. Fatores de riscos para o desenvolvimento de distúrbios alimentares: um estudo em universitárias. Rev Nutr. 2001;14(supl. 0):3-6.

27. Hsu LK. Epidemiology of the eating disorders. Psychiatr Clin North Am. 1996;19(4):681-700.

28. Morgan CM, Vecchiatti IR, Negrão AB. Etiologia dos transtornos alimentares: aspectos biológicos, psicológicos e sócio-culturais. Rev Bras Psiquiatr. 2002;24(supl. 3):18-23.

29. Sepulveda AR, Carrobles JA, Gandarillas AM. Gender, school and academic year differences among Spanish university students at high-risk for developing na eating disorder: na epidemiologic study. BMC Public Heath. 2008;8:102.

30. Hughes R, Desbrow B. Aspiring dietitians study: a pre-enrolment study of students motivations, awareness and expectations relating to careers in nutrition and dietetics. Nutr Diet. 2005;62(2-3):106-9.

31. Schmidt MI, Duncan BB, Tavares M, Polanczyk CA, Pellanda L, Zimmer PM. Validity of self-reported weight - A study of urban Brazilian adults. Rev Saude Publica. 1993;27(4):271-6.

32. Spencer EA, Appleby PN, Davey GK, Key TJ. Validity of self-reported height and weight in 4808 EPIC-Oxford participants. Public Health Nutr. 2002;5(4):561-5. 